\title{
Attraction of Plasmodia of the Myxomycete, Badhamia utricularis, by Extracts of the Basidiomycete, Stereum hirsutum
}

\author{
By M. F. MADELIN, FIONA AUDUS* AND D. KNOWLES $\dagger$ \\ Department of Botany, University of Bristol, Bristol BS8 I UG
}

(Received 25 November 1974; revised 25 March 1975)

\begin{abstract}
SUMMARY
Pieces of the fruit body of Stereum hirsutum attracted the migrating plasmodia of Badhamia utricularis at a distance of $4 \mathrm{~cm}$. Extracts of fruit bodies made with organic solvents could attract, but aqueous extracts could not. Cultured mycelium and extracts of cultured mycelium also attracted strongly, but activity was not detected in culture filtrate. Phase separation with organic and aqueous phases concentrated the attractive principle. Paper chromatography indicated the presence of a single substance of high activity which migrated in isopropanol-ammoniawater with an $R_{\mu}$ of 0.83 to 0.88 and in butanol-acetic acid-water with an $R_{F}$ close to 0.9 . The active extracts from fruit bodies and cultured mycelium were thermostable. The attractant diffused through both aqueous and gaseous phases.
\end{abstract}

\section{INTRODUCTION}

Lister (I 888) reported that the plasmodium of Badhamia utricularis Berkeley could feed upon a number of Basidiomycetes but that it preferred the fructification of Stereum hirsutum, over which it usually crawled in the course of a few hours. Reports of the attraction of myxomycete plasmodia by chemical substances are fairly numerous (Hawker, 1952; Ziegler, I962). Emoto (I932) reported that plasmodia of Physarum viride and Physarum rigidum were attracted by extracts of fruit bodies of eleven different Hymenomycetes. Carlile (1970) reported on the chemotactic effectiveness of sugars or peptone for plasmodia of Physarum polycephalum, and Konijn \& Koevenig (197I) on the attraction of myxamoebae and plasmodia of Didymium iridis and Physarum pusillum by Escherichia coli or a mixture of amino acids.

In the course of routine cultivation of plasmodia of $B$. utricularis on pieces of the fruit body of $S$. hirsutum we observed that the latter attracted plasmodia; we have investigated this phenomenon.

\section{METHODS}

Organisms and routine culture. Plasmodia of Badhamia utricularis were cultured either on oatmeal agar [6o g porridge oats (Quaker Oats Ltd, Southall, Middlesex), Io g agar, I 1 distilled water] or on $2 \%$ plain agar sprinkled with"a few oat flakes (Quaker porridge oats) in $9 \mathrm{~cm}$ diameter Petri dishes and incubated in darkness at room temperature (about $20^{\circ} \mathrm{C}$ ). After 2 days, plasmodia were transferred to Petri dishes containing only plain agar and 2 days later were used in experiments. Although other micro-organisms were not seen in these

\footnotetext{
* Present name and address: Mrs F. Pushman, Plant Breeding Institute, Trumpington, Cambridge.

$\dagger$ Present address: Department of Biochemistry, Imperial College of Science and Technology, London SW7 2 BB.
} 
cultures it is doubtful whether they were truly axenic. To produce sclerotia for storage, plasmodia which had advanced on to damp filter paper were dried by exposure in half-open Petri dishes for several hours.

Dikaryotic mycelium of Stereum hirsutum, isolated from spores from a fruit body from Ashton Court, Bristol, was grown on peptone-yeast extract-glucose agar (PYG agar), containing $(\mathrm{g} / \mathrm{l})$ : bacteriological peptone $(\mathrm{BDH}), \mathrm{I} \cdot 25$; yeast extract (Oxoid), $\mathrm{I} \cdot 25$; glucose, 3; agar, 20; glass-distilled water to I 1. Extracts of mycelium of $S$. hirsutum were obtained from batches of eight $500 \mathrm{ml}$ Erlenmeyer flasks, each containing $200 \mathrm{ml}$ PYG solution inoculated with pieces of mycelium from stock cultures and incubated on a rotary shaker ( $\mathrm{cm}$ radius throw, at $\mathrm{I} 20 \mathrm{rev} . / \mathrm{min}$ ) at approximately $20{ }^{\circ} \mathrm{C}$ for 7 days under subdued natural daylight illumination.

Fruit bodies of $S$. hirsutum used in experiments were collected from decaying logs near Bristol.

Paper chromatography. One-dimensional descending paper chromatography was used to fractionate extracts of Stereum mycelium. An acetone solution of extract ( $\mathrm{I} \mathrm{ml}$ ) was layered evenly on to Whatman's No. I chromatography paper and developed with either isopropanol-ammonia-distilled water (IAW) (IO:I:I, by vol.) or butanol-acetic acid-distilled water (BAW) (I2:3:5, by vol.) for $10 \mathrm{~h}$ at 20 to $22{ }^{\circ} \mathrm{C}$.

'Quadrant test' for detection of positive chemotactic effectiveness. Plastic Petri dishes ( $9 \mathrm{~cm}$ diameter), containing plain agar $(2 \%, \mathrm{w} / \mathrm{v}) \mathrm{I} \cdot 6$ or $2 \cdot 0 \mathrm{~mm}$ deep, were quartered by two diameters marked on the base of each dish. The material to be tested for chemotactic effectiveness was placed half-way along the margin of one of the sectors (the 'test sector'). A $5 \mathrm{~mm}$ square of filter paper bearing a piece of a Badhamia sclerotium or a similar-sized piece of plasmodium was placed at the centre of the plate. When pieces of plasmodium were used, their original fronts were faced in different directions with respect to the test sector in each of the several replicate plates which were prepared. Several replicate plates were incubated in darkness at room temperature for $\mathrm{I} 6 \mathrm{~h}$, unless otherwise specified. Each plasmodium had usually by then migrated away from its original site into one of the four sectors. If it had failed to move from its original site, that particular test was disregarded. The probability that plasmodia migrated into the test sector by chance was calculated from the equation:

$$
P=\left(\frac{1}{4}\right)^{x} \times\left(\frac{3}{4}\right)^{n-x} \times \frac{n !}{x !(n-x) !} \times \mathrm{IOO},
$$

where $P$ is the percentage probability, $n$ the number of replicate plates and $x$ the number of plasmodia entering test sectors.

\section{RESULTS}

\section{Chemotactic properties of Stereum hirsutum and of extracts}

It was clear from many trials, including the following, that the fruit body of Stereum hirsutum was able to attract plasmodia at a distance. Twenty quadrant test plates were prepared bearing pieces of plasmodium. 'Test sectors' in the ten control plates were arbitrarily designated, but in the ten treatment plates each contained a piece of fruit body of Stereum hirsutum, about $7 \mathrm{~mm}$ square. After $30 \mathrm{~h}$, all ten plasmodia in the dishes containing $S$. hirsutum were located in the test sectors $(P=0.000 \mathrm{\%} \%$ ). In the control series, three of the migrating plasmodia had divided in two, a not uncommon event in the absence of a source of attraction. Of the 13 resulting plasmodia, 3 had entered the designated test sector $(P=25.2 \%)$ and 3,3 and 4 , respectively, had entered the other three sectors. The attraction 
of plasmodia, in this and similar tests, over a distance of $4 \mathrm{~cm}$, indicated that a diffusible or even volatile chemotactic principle was involved. Other experiments showed that positive chemotaxis was best observed on plain agar: it was not demonstrable on oatmeal agar.

Attempts were made to extract the attractant from the Stereum fruit bodies. Batches of fresh fruit bodies $(25 \mathrm{~g})$ were chopped and ground thoroughly in a small volume of solvent, left for $\mathrm{I} h$, and then removed by filtration through glass fibre. The extract was made up to $25 \mathrm{ml}$ with filtered washings of the material. All manipulations were at room temperature. The solvents used were water, ethanol, chloroform, hexane, diethyl ether or petroleum ether. Portions $(0.2 \mathrm{ml})$ of these extracts were separately added to $5 \mathrm{~mm}$ diameter discs of Whatman No. I filter paper and dried beneath a jet of cool air. For controls, $0 \cdot 2 \mathrm{ml}$ volumes of fresh solvent were evaporated to dryness on the discs. In quadrant tests, positive chemotaxis was detected towards only the organic solvent extracts. There was none towards aqueous extract or discs to which solvents alone had been applied.

The attractive principle in fruit bodies was stable. It was extractable from fruit bodies which had been stored for a week in a deep-freeze. Petroleum ether and ethanol extracts stored at about $4{ }^{\circ} \mathrm{C}$ were still active after I week, and petroleum ether solutions were still active after $30 \mathrm{~min}$ at $60^{\circ} \mathrm{C}$. The attractant was also effective at high dilutions. Petroleum ether extract prepared as above was serially diluted, and $0.1 \mathrm{ml}$ samples were placed on $5 \mathrm{~mm}$ diameter filter-paper discs which were dried and assayed for chemotactic effectiveness by the quadrant test with pieces of Badhamia sclerotium. From 2-fold to 32-fold dilution, all of the migrant plasmodia in 17 separate tests entered the test sector. Even at dilutions of 64-, I 28-, 256-, 5I2- and I024-fold, the probabilities of obtaining the observed migration into the test sector by chance alone were less than $5 \%$ and so were regarded as significant. The use of greater dilutions than these led to no acceptably significant chemotactic responses.

The quadrant test was used to determine whether mycelial cultures of Stereum also possessed attractant activity. Test discs of Stereum colonies $(9 \mathrm{~mm}$ diameter) were excised from 7-day-old plate cultures on PYG agar, and control discs of the culture medium were cut from uninoculated PYG. Six replicate tests of each series were prepared. All six plasmodia reached the discs cut from the Stereum cultures $(P=0.02 \%)$, but in the controls only two of the six plasmodia entered the positive sector $(P=30 \%)$. It was concluded that the mycelial cultures had attractant activity.

Separate tests were made for the presence of attractant activity in mycelium and in medium from a batch of eight Stereum cultures grown in liquid medium. The mycelium was harvested by filtration through four layers of muslin, washed five times in tap water by repeated resuspension and filtration, coarsely chopped, and immersed in $15 \mathrm{ml}$ of a solvent as specified below for $\mathrm{I} h$ with occasional stirring. After filtration through glass wool, the filtrate was made up to $25 \mathrm{ml}$ with washings of the mycelium with pure solvent. Extracts of culture filtrate were prepared by phase separation of $250 \mathrm{ml}$ of culture filtrate with $25 \mathrm{ml}$ petroleum ether (b.p. 40 to $60^{\circ} \mathrm{C}$ ). The two phases were inverted five times and then left for $20 \mathrm{~min}$ before separating them. Subsequent phase separations with various solvents, as detailed below, were done with equal volumes of the two phases. To test for attractant activity in organic solvents, $0.2 \mathrm{ml}$ samples of solution were evaporated to dryness on $5 \mathrm{~mm}$ diameter discs of filter paper which were then used in quadrant tests. Aqueous solutions were tested by adding enough to fill $8 \mathrm{~mm}$ diameter wells cut in the test sectors. Both acetone and petroleum ether extracts of mycelium were of high chemotactic effectiveness, all plasmodia migrating on to the sources of attractant $(P=0.02$ and $0.1 \%$, respectively). When acetone and petroleum ether extracts of mycelium each were phase-separated with the other solvent, detectable attractant activity then resided wholly or chiefly in the acetone 
phase. No activity was detected in the aqueous culture filtrate, or in petroleum ether phase separations of this, or in the residual aqueous phase.

Although the aqueous extract of fruit bodies, the aqueous culture filtrate, and the aqueous phase from separations against organic solvents possessed no demonstrable attractant activity, it was nevertheless possible to transfer high activity to aqueous solutions if an acetone extract was phase-separated against petroleum ether, the acetone phase evaporated to dryness, and the residue shaken vigorously with water. In eight quadrant test plates, the plasmodia all entered the test sector $(P=0.002 \%)$ and seven contacted the source. If the phase separation against petroleum ether was omitted, the final activity was very much reduced and of doubtful significance $(P=9 \%)$. Possibly the petroleum ether served to remove lipophilic substances which otherwise prevented the attractant(s) in the dry residue from dissolving in water.

On the basis of the above experiments, the procedure adopted as standard for producing a highly active extract from harvested mycelium of Stereum was to grind it in a mortar with Celite (BDH), extract it with acetone as described, and phase separate it with petroleum ether, the consequent acetone fraction constituting the desired preparation.

The thermostability of the attractant from Stereum mycelium was tested by heating samples of an aqueous solution, to which attractant activity had been transferred by the procedure described above, at $80{ }^{\circ} \mathrm{C}$ for $30 \mathrm{~min}$ and at I I ${ }^{\circ} \mathrm{C}$ (under pressure, in an autoclave) for $20 \mathrm{~min}$, and testing residual activity by means of quadrant tests each with six replicates. All six plasmodia in each test migrated towards the wells containing the heattreated solutions $(P=0.024 \%)$. Like the extract from Stereum fruit bodies, extract from mycelium was thermostable.

\section{Partial purification of attractant from mycelium of Stereum hirsutum}

Acetone extracts of Stereum mycelium were prepared by the standard method (above) and fractionated by paper chromatography with the solvent systems IAW and BAW. To determine the presence and distribution of attractant activity on longitudinal strips cut from descending chromatograms, the 'clock test' was developed. A $9 \mathrm{~cm}$ diameter dish containing $2 \%$ plain agar $\mathrm{I} \cdot 6 \mathrm{~mm}$ deep was divided into eight equal sectors by marks on the exterior of the dish, and a plasmodial inoculum was placed at its centre. An entire longitudinal strip, $5 \mathrm{~mm}$ wide, cut from the chromatogram, was divided into a series of equal lengths numbered I to 8 from the origin to the solvent front. The pieces were placed in sequence around the periphery of the agar plate, one per sector. Eight replicates, each using a complete longitudinal strip from the same IAW-developed paper chromatogram, were prepared in each experiment. To avoid bias, the central plasmodial inoculum was placed so that its original forward direction was aimed at a differently numbered sector in each replicate. After incubation for $\mathrm{I} 6 \mathrm{~h}$, in darkness at room temperature, the particular sectors containing the migrating front of each plasmodium were noted together with the numbers of plasmodia which had actually contacted the strips. The plasmodium failed to migrate in one replicate test which was therefore disregarded. Six plasmodia entered sector No. 7 (corresponding to $R_{F} 0.75$ to 0.88 ), four of them contacting the corresponding paper strips; and one entered sector No. $6\left(R_{F} 0.63\right.$ to 0.75$)$. The probability of six out of eight plasmodia entering the same sector by chance, calculated by a method analogous to that used for quadrant tests, is $0.008 \%$.

The position of the attractant was located more precisely by another clock assay with strips of the same chromatogram corresponding to $R_{F} 0.5$ to $\mathrm{r} \cdot 0$, each divided into eight pieces. Five replicate tests were prepared. In three the plasmodium had migrated on to the 
strips corresponding to $R_{F} 0.83$ to 0.88 , in the fourth it was still migrating towards this region, while in the fifth it had failed to move from its inoculation site. The probability of all four migrating plasmodia entering the same eighth of the clock test by chance was $<0.025 \%$.

The results of similar tests on a chromatogram developed with BAW showed that the activity was principally in the region above $R_{F} 0.625$. In the light of this result, three independently prepared extracts of mycelium were chromatographically developed with BAW and assayed by the clock method, but with only the distal third of each strip of chromatogram $\left(R_{F} 0.67\right.$ to $\left.\mathrm{I} \cdot 0\right)$ cut into eight pieces for the test. A total of 20 replicate tests was performed. In two the plasmodia divided whilst migrating, and in one the plasmodium failed to migrate; these were disregarded. In the remaining 17 tests one plasmodium entered the sector corresponding to $R_{F} 0.79$ to 0.83 , four entered $R_{F} 0.83$ to 0.88 , nine entered $R_{F} 0.88$ to 0.92 , and three entered $R_{F} 0.92$ to 0.96 . Ten of these plasmodia actually contacted the strips of paper. The probability of nine out of seventeen migrating plasmodia entering a single sector $\left(R_{F} 0.88\right.$ to 0.92$)$ by chance was $0.006 \%$. Thus most of the attractant activity lay between $R_{F} 0.83$ and 0.96 and was apparently centred upon $R_{F} 0.90$.

Because it is possible that the results of clock tests could be affected by negative chemotactic agents or other interfering substances elsewhere on the peripheral strips of chromatogram, the above results were checked by cutting $0.5 \mathrm{~cm}$ wide strips from a chromatogram developed with BAW into I 6 successive pieces from the origin to the front, and using the pieces in quadrant tests of chemotactic effectiveness prepared in triplicate. With the third, thirteenth and fourteenth pieces the plasmodia entered the test sector in two of the three replicates $(P=14 \%)$, but only with the fifteenth piece were all three of the replicates positive $(P=1 \cdot 6 \%)$, and in two of these the plasmodium actually contacted the paper. The fifteenth piece corresponded to an $R_{F}$ of 0.88 to 0.94 . The slight possibility that a subsidiary attractant had migrated to the third piece $\left(R_{F} 0.13\right.$ to 0.18$)$ was not confirmed in other tests that were done.

\section{Investigation of the volatility of attractant from Stereum}

Numerous tests revealed a rapid initiation of the chemotactic response to the attractant, which suggested that the attractant was of low molecular weight and perhaps volatile. To determine whether it diffused through the agar substrate or the atmosphere or both, quadrant tests were employed. In one experiment the paper disc on which $0.2 \mathrm{ml}$ of standard extract of Stereum mycelium had been dried was placed on aluminium foil just within the margin of a Petri dish containing $20 \mathrm{ml}$ of plain agar, so as to prevent diffusion of attractant via the agar. In a second experiment the disc was placed directly on a layer of $\mathrm{I} 0 \mathrm{ml}$ of plain agar as usual, but was immediately covered by another layer of $10 \mathrm{ml}$ of gelled plain agar transferred from another Petri dish. Attractant could therefore leave the sandwiched disc only by diffusion through the aqueous phase in the agar. Two control treatments were similar to the above except that the discs were not impregnated with Stereum extract. Five replicate plates of each treatment were incubated for $20 \mathrm{~h}$ at $20^{\circ} \mathrm{C}$. In each treatment with attractant present, all five plasmodia had entered the test sectors of the quadrant plates $(P=0.1 \%)$ and also had either contacted the foil tray or migrated on top of the sandwiched disc. In one of the control series, one plasmodium had entered a test sector $(P=40 \%)$ but had not closely approached the disc. In the other control series, no plasmodia had entered the test sectors $(P=24 \%)$. It is evident that the attractant could traverse both gaseous and aqueous phases. 


\section{DISCUSSION}

Plasmodia of $B$. utricularis showed positive chemotaxis towards a constituent of $S$. hirsutum. There was no evidence that fruit bodies and cultured mycelium of $S$. hirsutum contained different attractants.

The attractant was highly active and volatile. A more than I0oo-fold dilution of fruit-body extract prepared as described was able to attract plasmodia over a distance of about $4 \mathrm{~cm}$ in quadrant tests. The amount of attractant actually present in such a test corresponded to I $00 \mu \mathrm{g}$ fresh weight of $S$. hirsutum fruit body. The effectiveness of the attractant at such low concentrations suggests it acts differently from the sugars (including glucose) and peptone which Carlile (1970) reported to be chemotactically effective for Physarum polycephalum at $\mathrm{I} \%(\mathrm{w} / \mathrm{w})$ concentrations; Coman (I940) had found glucose to be effective with $P$. polycephalum from $0 \cdot 0$ I to I $\mathrm{M}$ (i.e. $0 . \mathrm{I} 8$ to $\mathrm{I} 8 \%$, w/v). Carlile found a complete correlation between the ability of sugars to support growth and their chemotactic effectiveness. It is likely that the attractant from $S$. hirsutum is not a nutrient but instead is a substance which specifically affects plasmodial migration, even though its activity is affected by nutrients as shown by the absence of the effect on oatmeal agar.

We thank Miss Margaret Pace and Dr M. H. Martin for helpful advice on statistical matters.

\section{REFERENCES}

Carlile, M. J. (1970). Nutrition and chemotaxis in the myxomycete Physarum polycephalum: the effect of carbohydrates on the plasmodium. Journal of General Microbiology 63, 22 I-226.

Coman, D. R. (1940). Additional observations on positive and negative chemotaxis: experiments with a myxomycete. Archives of Pathology 29, 220-228.

Емото, Y. (1932). Über die Chemotaxis der Myxomyceten-Plasmodien. Proceedings of the Imperial Academy of Japan 8, 460-463.

Hawker, L. E. (1952). The physiology of Myxomycetes. Transactions of the British Mycological Society 35 , I77-187.

KoniJn, T. M. \& KoeveniG, J. L. (I97I). Chemotaxis in Myxomycetes or true slime molds. Mycologia 63, $90 \mathrm{I}-906$.

Lister, A. (I888). Notes on the plasmodium of Badhamia utricularis and Brefeldia maxima. Annals of Botany 2, I-24.

Ziegler, H. (1962). Chemotaxis. In Encyclopedia of Plant Physiology, vol. I7, part 2, pp. 484-532. Edited by W. Ruhland. Berlin: Springer. 